\title{
Paronychia and Periungual Granulation as a Novel Side Effect of Ibrutinib: A Case Report
}

\author{
Ahu Yorulmaz Basak Yalcin \\ Department of Dermatology, Ankara Bilkent City Hospital, Ankara, Turkey
}

\section{Established Facts}

- Targeted therapies have nail-related side effects.

- Ibrutinib is a targeted agent that is effective in the treatment of chronic lymphocytic leukaemia and B-cell malignancies.

\section{Novel Insights}

- Brittle nails is the most commonly reported nail-related side effect of ibrutinib.

- Paronychial inflammation and periungual granulation are newly reported nail-related side effects of ibrutinib.

\section{Keywords}

Ibrutinib · Paronychia - Periungual granulation - Side effect

\begin{abstract}
Ibrutinib is an oral covalent inhibitor of the Bruton's tyrosine kinase pathway and is approved for the treatment of B-cell malignancies including chronic lymphocytic leukaemia, mantle cell lymphoma, and Waldenström's macroglobulinaemia. It is generally a drug of choice for high-risk patients with indolent lymphomas. The safety profile of ibrutinib appears to be tolerable, with well-known side effects such as infections and haematologic complications. Additionally, dermatological adverse reactions with ibrutinib therapy have been reported to encompass maculopapular rash and hair/nail abnormal-
\end{abstract}

\section{KARGER}

(c) 2019 S. Karger AG, Basel

E-Mail karger@karger.com

www.karger.com/sad ities. Here, we present a case of ibrutinib-induced paronychia and periungual granulation in a 40-year-old woman. To the best of our knowledge, this is the third description of ibrutinib-induced paronychia and periungual granulation.

(c) 2019 S. Karger AG, Basel

\section{Introduction}

Ibrutinib is an oral tyrosine kinase inhibitor that irreversibly binds and inhibits Bruton's tyrosine kinase (BTK). Targeting BTK with ibrutinib has been shown to be effective in treating B-cell malignancies such as chronic lymphocytic leukaemia (CLL), mantle cell lymphoma, Waldenström's macroglobulinaemia, diffuse large B-cell lymphoma, follic- 


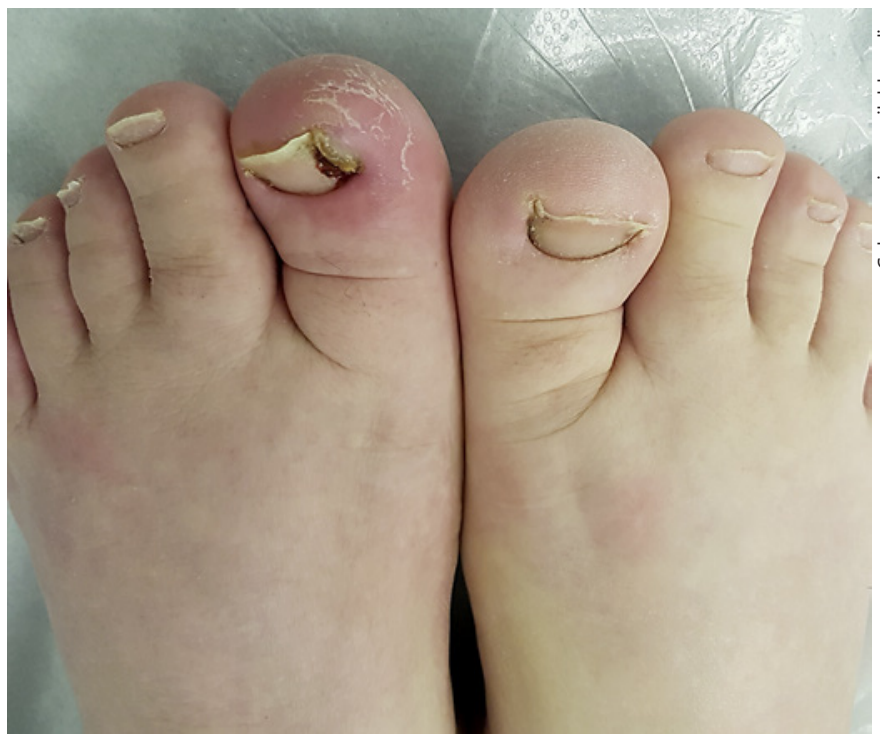

Fig. 1. Enlarged feet.

ular lymphoma, and multiple myeloma. Ibrutinib has a favourable side effect profile, although as with other molecular targeted therapies, it may also induce toxicities that are distinct from those of classical immunochemotherapy [1, 2]. Here, we report a case of ibrutinib-induced paronychia and periungual granulation. To the best of our knowledge, this is the third case in the literature [3] describing ibrutinib-induced paronychia and periungual granulation.

\section{Case Report}

A 40-year-old woman visited our outpatient clinic with a 2-month history of swelling and redness in her left big toe. She had been diagnosed with CLL 5 years earlier, for which she had been under ibrutinib for the last 2 years. Moreover, her medical history revealed acromegaly, type 2 diabetes mellitus, and hypothyroidism. Her medication history included subcutaneous insulin injection, metformin (500 mg twice daily), pegvisomant (60 mg weekly), levothyroxine ( $75 \mu \mathrm{g}$ daily), and intravenous immunoglobulin infusion (every 2 months). She reported similar complaints in her right big toe 6 months ago, which healed within months. She denied any history of trauma preceding the findings. A dermatological examination revealed enlarged feet with an erythematous and swollen left big toe and a serosanguinous discharge on its lateral nail fold (Fig. 1, 2). Laboratory investigations, including complete blood and differential counts, erythrocyte sedimentation rate, and C-reactive protein levels, were within normal ranges. Moreover, a bacterial wound culture was conducted that yielded negative results. Thus, based on the history and clinical and laboratory findings, a diagnosis of ibrutinib-induced paronychia and periungual granulation was established. The patient was prescribed boric acid soak and topical corticosteroids; however, she was lost to follow-up.

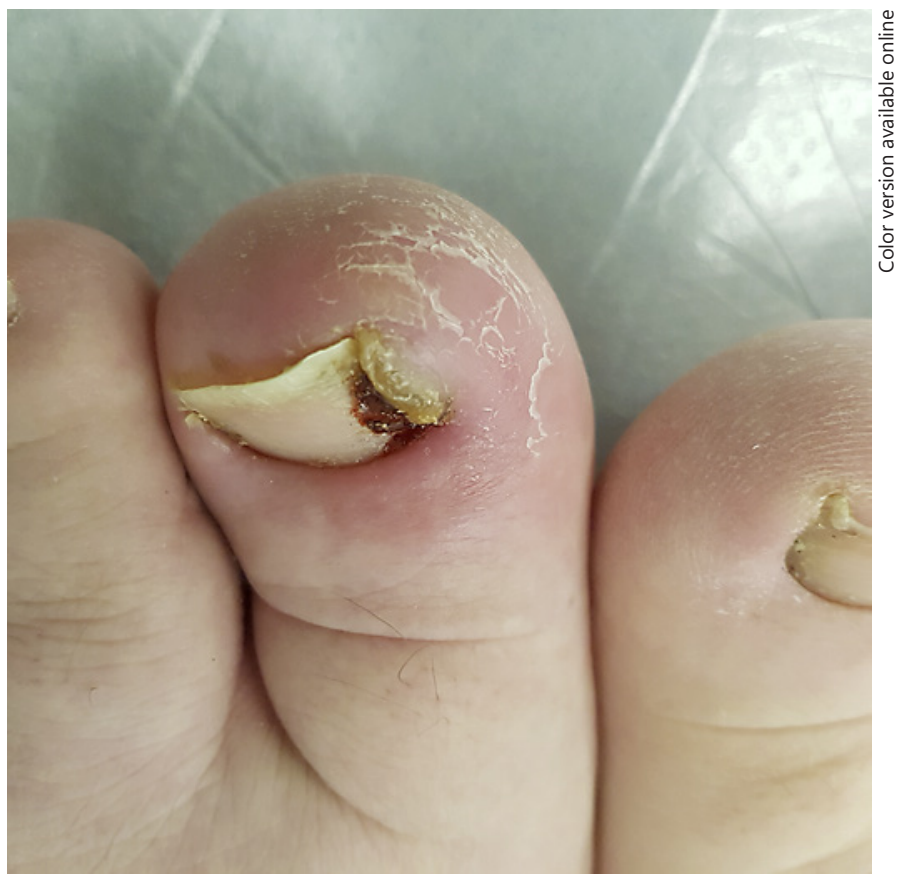

Fig. 2. Erythema and swelling in the left big toe. Paronychia characterized by serosanguinous discharge, crusting, and granulation tissue of lateral nail fold.

\section{Discussion}

Molecular targeted therapies have revolutionised cancer treatments. These therapies directly and specifically target cancer cells or specific signalling molecules/ pathways necessary for the growth of cancer cells. These are potentially well tolerated; however, it has been reported that molecular targeted therapies have side effects that are not observed with the conventional immunochemotherapy-based regimens [4]. In recent years, a growing number of reports have highlighted nail-related side effects of targeted therapies. For instance, paronychia and periungual pyogenic granuloma are welldocumented nail-related toxicities of epidermal growth factor receptor inhibitors (EGFRIs), epidermal growth factor receptor tyrosine kinase inhibitors, mitogen-activated protein kinase inhibitors, and mammalian target of rapamycin inhibitors [5-9] (Table 1). As a targeted therapy, ibrutinib has major side effects such as infections, haematological complications, and cardiac adverse reactions $[1,2,10]$. Up to now, only a single report is available [3] on the most common nail toxicity of targeted therapies, which is paronychia and periungual granulation. However, recently, there have been few re- 
Table 1. Nail-related side effects of other targeted therapies

\begin{tabular}{ll}
\hline Drug & Nail-related side effect \\
\hline EGFRIs & Paronychia, periungual granuloma, onycholysis, brittle nails, slower nail growth rate, \\
& nail dystrophy, dyspigmentation [5, 7-9, 20] \\
EGFR-TKIs & Paronychia, periungual granuloma [6, 20] \\
MEKIs & Paronychia, periungual granuloma, onycholysis, brittle nails, slower nail growth rate [5, 20] \\
mTORi & Paronychia, periungual granuloma, onycholysis, brittle nails, slower nail growth rate [5] \\
Selective pan-FGFR 1-4 inhibitors & Onycholysis, onychomadesis, nail bed superinfection [5] \\
BRAFi & Onycholysis, brittle nails, paronychia [7, 20] \\
VEGFI & Paronychia [20] \\
Anti-CD20 monoclonal antibody & Periungual granuloma [20] \\
TKIs & Periungual granuloma [20] \\
\hline
\end{tabular}

EGFRIs, epidermal growth factor receptor inhibitors; EGFR-TKIs, epidermal growth factor receptor tyrosine kinase inhibitors; MEKIs, mitogen-activated protein kinase inhibitors; mTORi, mammalian target of rapamycin inhibitors; pan-FGFR, pan-fibroblast growth factor receptor; VEGFI, vascular endothelial growth factor inhibitors; TKIs, tyrosine kinase inhibitors.

ports describing nail-related side effects associated with ibrutinib therapy [10-13].

Bitar et al. [13] investigated hair and nail manifestations associated with the long-term use of ibrutinib in 66 patients with CLL. They found that $67 \%$ of the patients reported brittle fingernails, while $23 \%$ reported brittle toenails. Nail abnormalities commonly appeared several months after initiating ibrutinib. Similarly, Heldt Manica and Cohen [12] reported the development of brittle nails in 2 patients after 4 and 6 months, respectively, after initiating ibrutinib treatment. Thus, according to these reports, nail abnormality related to ibrutinib therapy is a common cutaneous toxicity. However, this reported toxicity includes only the brittle nails. We aimed to highlight newly documented side effects of ibrutinib, i.e., paronychial inflammation and periungual granulation. These were first reported by Mohandas and Davis [3] in 2 patients, and to the best of our knowledge, this is the third report of this novel side effect of ibrutinib.

According to the literature, targeted therapies are known to induce nail toxicities, with paronychia and periungual granulomas as the most commonly reported side effects. Paronychia and periungual granulomas are generally documented in response to EGFRIs, with an incidence of $17.2 \%$ of all-grade nail toxicity $[5,14]$. Paronychial inflammation of the toes or fingers begins within weeks or months after initiating drugs and is initially characterised by erythema, oedema, and tenderness of the nail folds. Periungual inflammatory paronychia gradually evolves as an overgrowth of the friable granulation tissue, which is a pyogenic granuloma-like lesion. Although, the whole process mimics the progres- sion of an ingrown nail, the pathophysiology underlying targeted therapy-associated nail toxicities is different, with the mechanism being inflammatory in nature $[5,6$, 14].

Mohandas and Davis [3] highlighted that ibrutinib is associated with excessive granulation tissue formation with painful inflammation of the lateral nail folds consistent with paronychia. This finding was associated with inflammation as no other medication, traumatic trigger, or laboratory findings supportive of infections was found in the patients. Moreover, systemic antibacterial/antifungal treatments were ineffective, and lesional skin biopsy was consistent with perivascular lymphohistiocytic inflammation with neutrophils [3]. Similarly, we favoured the diagnosis based on the literature and individual patient data and specified the features of this side effect (Table 2).

The exact mechanism underlying in the development of this side effect is unclear. It is well-known that retinoids have the potential to cause paronychia, brittle nails, excess granulation tissue, and pyogenic granuloma formation [15]. Baran et al. [16] suggested that desquamative dermatitis is the primary mechanism involved in the development of retinoid-related nail side effects. Desquamation is usually accompanied by the thinning of the epidermis and its extent is proportional with the severity of nail fragility. Meanwhile, with the accumulation of scales in the nail folds, desquamative phenomenon may act as a foreign body in the lateral nail groove, leading to inflammation and the formation of granulation tissue. In addition, nail fragility results in fine spicules that break through the lateral nail grooves and induce the formation of granulation tissue and ingrown nails [16].
Yorulmaz/Yalcin 
Table 2. Summary of ibrutinib-induced nail toxicity in the literature

\begin{tabular}{|c|c|c|c|c|c|}
\hline Author(s) & Article type & Study characteristics & Results & Patient characteristics & Presentation \\
\hline Bitar et al. [13] & $\begin{array}{l}\text { Prospective } \\
\text { observational } \\
\text { study }\end{array}$ & $\begin{array}{l}\text { Included } 66 \text { patients } \\
\text { with CLL treated with } \\
\text { ibrutinib }\end{array}$ & $\begin{array}{l}n=44(67 \%) \\
\text { developed brittle } \\
\text { fingernails, } n=15 \\
\text { (23\%) developed } \\
\text { brittle toenails }\end{array}$ & & \\
\hline $\begin{array}{l}\text { Mohandas } \\
\text { and Davis [3] }\end{array}$ & Case report & - & - & $\begin{array}{l}79 \text {-year-old male } \\
\text { with mantle cell } \\
\text { lymphoma }\end{array}$ & $\begin{array}{l}\text { Excessive } \\
\text { granulation tissue } \\
\text { around multiple } \\
\text { toenails with } \\
\text { paronychial } \\
\text { inflammation of the } \\
\text { lateral nail folds } \\
\text { Inflammation around } \\
\text { the cuticle of the left } \\
\text { index finger }\end{array}$ \\
\hline
\end{tabular}

We postulate that the mechanism involved in the pathogenesis of ibrutinib-induced nail abnormalities is analogous to that of retinoid-related nail abnormalities. Ibrutinib is an irreversible tyrosine kinase inhibitor that covalently bonds with a cysteine residue on BTK. As cysteines are crucial for nail integrity, ibrutinib-induced disruption of the disulphide bonds between cysteine residues may serve as the main factor in nail fragility [13]. Nail fragility leads to desquamative foci that are reserved at the under surface of the proximal nail fold and eventually act as foreign bodies and trigger an inflammatory response [16].

Furthermore, we highlight another possible mechanism underlying ibrutinib-induced paronychia and periungual pyogenic granuloma-like lesions. As BTK mediates nuclear factor $-\kappa B(N F-\kappa B)$ activation, ibrutinib in turn inhibits NF- $\kappa B$ signalling by inhibiting BTK [17]. $\mathrm{NF}-\kappa \mathrm{B}$ is expressed in all cell types and tissues, and NF- $\mathrm{KB}$ signalling is implicated in several steps of carcinogenesis, including pathogenic stimulus, chronic inflammation, fibrosis, establishment of remodelling, and transition of normal cells to cancer cells. Various inflammatory cytokines activate NF- $\kappa B$, leading to the stimulation of cell proliferation and inhibition of apoptosis [18]. Although the NF- $\kappa \mathrm{B}$ pathway has long been considered as a prototypical proinflammatory signalling pathway, recent evidence has revealed both its pro- and anti-inflammatory

Nail Toxicities of Ibrutinib characteristics [19]. We suggest that ibrutinib-induced paronychia and periungual granulation represent an inflammatory response, involving various cytokines, chemokines, and NF- $\mathrm{KB}$ signalling that lead to chronic inflammation, fibrosis, and establishment of remodelling. Although the downregulation of the NF- $\mathrm{BB}$-mediated pathways is generally expected to result in the inhibition of inflammation and cell proliferation, there may be another NF- $\kappa \mathrm{B}$ pathway-related mechanism contributing to the development of this novel side effect of ibrutinib.

Herein, we have reported a case of ibrutinib-induced paronychia and periungual granulation as a novel side effect. We assume that this side effect is associated with nail kinetics and morphology. As new drugs are on the horizon, novel side effects are certain to emerge. Awareness regarding these side effects is crucial to avoid misdiagnosis and unnecessary diagnostic procedures and treatments, especially in high-risk patients.

\section{Statement of Ethics}

The patient's consent was obtained.

\section{Disclosure Statement}

None of the authors declare any financial support or relationships that may pose a conflict of interest regarding this paper. 


\section{References}

1 Schwarzbich MA, Witzens-Harig M. Ibrutinib. Recent Results Cancer Res. 2014;201: 259-67.

2 de Weerdt I, Koopmans SM, Kater AP, van Gelder M. Incidence and management of toxicity associated with ibrutinib and idelalisib: a practical approach. Haematologica. 2017 Oct; 102(10):1629-39.

3 Mohandas D, Davis M. Paronychia and excess granulation tissue with ibrutinib: a newly reported side effect. J Am Acad Dermatol. 2016;74(5):AB222.

4 Padma VV. An overview of targeted cancer therapy. Biomedicine (Taipei). 2015 Nov; 5(4): 19 .

5 Lacouture M, Sibaud V. Toxic Side Effects of Targeted Therapies and Immunotherapies Affecting the Skin, Oral Mucosa, Hair, and Nails. Am J Clin Dermatol. 2018 Nov;19 (Suppl 1):31-9.

6 Dainichi T, Tanaka M, Tsuruta N, Furue M, Noda K. Development of multiple paronychia and periungual granulation in patients treated with gefitinib, an inhibitor of epidermal growth factor receptor. Dermatology. 2003; 207(3):324-5
7 Dika E, Patrizi A, Ribero S, Fanti PA, Starace M, Melotti B, et al. Hair and nail adverse events during treatment with targeted therapies for metastatic melanoma. Eur J Dermatol. 2016 Jun;26(3):232-9.

8 Cubero DI, Abdalla BM, Schoueri J, Lopes FI, Turke KC, Guzman J, et al. Cutaneous side effects of molecularly targeted therapies for the treatment of solid tumors. Drugs Context. 2018 Jul;7:212516.

9 Macdonald JB, Macdonald B, Golitz LE, LoRusso P, Sekulic A. Cutaneous adverse effects of targeted therapies: Part I: Inhibitors of the cellular membrane. J Am Acad Dermatol. 2015 Feb;72(2):203-18.

10 Paydas S. Management of adverse effects/toxicity of ibrutinib. Crit Rev Oncol Hematol. 2019 Apr;136:56-63.

11 Levy I, Polliack A, Tadmor T. Five IbrutinibAssociated Side Effects That All Clinicians Should Be Aware of. Acta Haematol. 2019; 141(4):254-5.

12 Heldt Manica LA, Cohen PR. Ibrutinib-Associated Nail Plate Abnormalities: Case Reports and Review. Drug Saf Case Rep. 2017 Nov; 4(1): 15 .

13 Bitar C, Farooqui MZ, Valdez J, Saba NS, Soto S, Bray A, et al. Hair and Nail Changes During Long-term Therapy With Ibrutinib for Chronic Lymphocytic Leukemia. JAMA Dermatol. 2016 Jun;152(6):698-701.
14 Garden BC, Wu S, Lacouture ME. The risk of nail changes with epidermal growth factor receptor inhibitors: a systematic review of the literature and meta-analysis. J Am Acad Dermatol. 2012 Sep;67(3):400-8.

15 Figueiras DA, Ramos TB, Marinho AK, Bezerra MS, Cauas RC. Paronychia and granulation tissue formation during treatment with isotretinoin. An Bras Dermatol. 2016 Apr; 91(2):223-5.

16 Baran R. Etretinate and the nails (study of 130 cases) possible mechanisms of some side-effects. Clin Exp Dermatol. 1986 Mar;11(2): $148-52$.

17 Shinners NP, Carlesso G, Castro I, Hoek KL, Corn RA, Woodland RT, et al. Bruton's tyrosine kinase mediates NF-kappa B activation and $B$ cell survival by $B$ cell-activating factor receptor of the TNF-R family. J Immunol. 2007 Sep;179(6):3872-80.

18 Brücher BL, Lang F, Jamall IS. NF- $\kappa B$ signaling and crosstalk during carcinogenesis. 4open. 2019 May;2:13.

19 Lawrence T. The nuclear factor NF-kappaB pathway in inflammation. Cold Spring Harb Perspect Biol. 2009 Dec;1(6):a001651.

20 Wollina U. Systemic Drug-induced Chronic Paronychia and Periungual Pyogenic Granuloma. Indian Dermatol Online J. 2018 SepOct;9(5):293-8. 